\title{
CLÁUSULAS RELATIVAS EN EL INGLÉS CRIOLLO DE COSTA RICA
}

\author{
Mario Portilla
}

\begin{abstract}
RESUMEN
En el presente trabajo, se presenta una descripción de la tipología de la cláusula relativa del inglés criollo de Costa Rica, desde una perspectiva tipológico-funcional. Además, se estudian las distintas estrategias de representación de la frase nominal relativizada y la jerarquía de las relaciones gramaticales en esta lengua.

Palabras clave: cláusula relativa, inglés criollo de Costa Rica.
\end{abstract}

\begin{abstract}
This article describes the types of relative clauses that occur in the English-based Creole of Costa Rica, according to a functional-typological approach. Furthermore, the different strategies used to express the relativized noun phrase and the by Keenan \& Comrie (1979) proposed hierarchy of grammatical relations are studied.

Key words: relative clause English-based of Costa Rica
\end{abstract}

\section{Introducción}

El presente trabajo pretende describir las oraciones relativas desde una perspectiva tipológico-funcional, tal como ha sido expuesta especialmente por Givón (1985 y 1990) y Payne (1997).

Dr. Mario Portilla Chaves. Profesor de la Escuela de Filología, Lingüística y Literatura de la Universidad de Costa Rica. San Pedro, San José, Costa Rica.

Correo electrónico: marioportilla2000@yahoo.com

Recepción: 01-07-2005

Aceptación: 02-07-2005 


\section{Clases de cláusulas relativas}

Una definición generalmente aceptada de las oraciones relativas es que son cláusulas que funcionan como modificadores nominales.

La función pragmática de las cláusulas relativas es ofrecer información que es conocida por el oyente o accesible a él, o que se supone que no es conocida por este ni accesible a él (cf. Givón 1990: 645 y ss.).

Por esta razón, tipológicamente se pueden distinguir dos tipos de cláusulas relativas:

\section{a. Cláusulas relativas restrictivas}

Las cláusulas restrictivas tienen la función de especificar o determinar el elemento de la cláusula principal que están modificando con información conocida por el oyente o que es accesible a él.

(1) A get di buk [we yu sen mi].

Yo recibir el libro REL vos mandar me

Recibi el libro que me mandaste.

(2) Mi de a tink pan [we yu se].

Yo DUR PRO pensar en REL vos decir

He estado pensando en lo que dijiste.

(3) Dem de kyar [we krash] waz a kom faas faas.

Ellos ahí carro REL chocar ANT PRO venir rápido rápido

Esos carros que chocaron venían muy rápido.

(4) Di ruod [we dem jos mek] no gud atáal.

La carretera REL ellos apenas hacer no ser-buena del-todo

La carretera que acaban de hacer no sirvió para nada.

En las cláusulas relativas (1) y (2), los contenidos que se trasmiten es información es conocida por el oyente, puesto que remite a acciones realizadas por este: el oyente envió el libro, el oyente dijo algo.

En (3) y (4), aunque la información no necesariamente es conocida por el oyente, esta parece ser accesible a él: el oyente puede observar el choque, el oyente puede ver la carretera.

Por otra parte, es importante resaltar que las cláusulas restrictivas, precisamente, restringen el significado del núcleo nominal que están modificando. Así, por ejemplo, el referente de la palabra MIIT carne, es menos extenso en (5) que en (6). La cláusula relativa en (5) permite especificar que no se trata de la carne en general, sino de la carne cocinada.

(5) Di miit [we kuk] no gud.

La carne REL cocinar no ser-buena

La carne que cocinaron no está buena. 
(6) Di miit no gud.

La carne no ser-buena

La carne no es buena

Prototípicamente, las relativas restrictivas son introducidas por el relativador wE, que es un pronombre. Además, estas cláusulas pueden ser introducidas mediante otras estrategias, como el uso del relativizador DAT (7) y (8) o la simple yuxtaposición de la relativa junto al núcleo nominal antecedente (9) y (10).

(7) Mi laas di buk [dat yu gi mi].

Yo perder el libro REL usted dar me

Perdi el libro que usted me dio.

(8) Di ous [dat wi si] mi no laik ${ }^{1}$.

La casa REL yo ver yo no gustar

La casa que vimos (no otra) no me gustó.

(9) Di píipl [mi a luk fa] néva de.

La gente yo PRO mirar por nunca COP-EXI

La gente que ando buscando nunca está.

(10) Di pícho [wi go si] di gud, man.

La película nosotros ir ver ANT ser-buena, hombre

La película que fuimos a ver estuvo buenísima.

\section{b. Cláusulas relativas no restrictivas}

La función de las cláusulas relativas no restrictivas no es especificar o determinar el núcleo nominal, sino más bien ofrecer una explicación adicional acerca de este núcleo. Esta aserción parentética contiene información que es considerada menos central dentro de la temática del discurso. Además, en contraposición con las cláusulas relativas restrictivas, el contenido de las relativas no restrictivas tiende a no estar presupuesto en el discurso. No se supone que la información sea conocida por el oyente o sea accesible a él (Givón 1990: 649).

(11) Jaan, [uu di se laik mi], gaan wid anáda úman.

J. REL ANT ${ }^{2}$ decir gustar me irse con otra mujer

John, quien decía quererme, se fue con otra mujer.

(12) Míeri, [uu mi waan mári], jos graduíet.

M. REL yo querer casar apenas graduar

Mary, con quien me quiero casar, acaba de graduarse.

(13) Mi ónkl, [we se av lat móni], taid.

Mi tío REL decir tener mucho dinero ser-tacaño

Mi tío, que dice que tiene mucha plata es un tacaño. 
(14) Airíin, [we véri relíjos], liv a chorch évri die.

A. REL muy ser-religioso vivir en iglesia cada día

Irene, que es muy religiosa, vive en la iglesia todos los días.

En primer lugar, hay que señalar que la cláusula relativa no restrictiva se encuentra separada del la cláusula principal por medio de pausas. Estas pausas están representadas por medio de comas. Además, el nivel de la curva de entonación de la relativa es más alto que el de la cláusula principal. Por esta razón, la cláusula relativa presenta un tonema ascendente al principio y uno descendente al final.

En segundo lugar, las cláusulas relativas no restrictivas son introducidas solamente por los pronombres relativos UU y WE. El primero parece utilizarse más prototípicamente que el segundo en este tipo de cláusulas.

Por último, es importante señalar que la información parentética propia de las cláusulas no restrictivas puede también ser introducida por medio de cláusulas coordinadas. Estas cláusulas relativas no se unen a la oración principal a través de una estrategia de subordinación, sino por medio de una estrategia de coordinación o parataxis. Este tipo de estrategia de relativización parece ser bastante común en las lenguas del mundo (Givón, 1990: 652) y, según Romaine (1988: 241 y ss.), es muy propia de los pidgins y aparece en los primeros estadios de las lenguas criollas.

En el criollo inglés de Costa Rica, el uso de esta estrategia de relativización no es frecuente. Sin embargo, ocurre ocasionalmente como se observa en (15).

Daríin ya $^{3}$, [shi mi áanti], kom fram Nyu Yaak yéside.

D. aquí ella mi tía venir de N.Y. ayer.

Esta Doreen, que es tía mía, vino de Nueva York ayer.

Una característica de este tipo de estrategia es que la correferencialidad entre el núcleo nominal y la frase nominal relativizada se expresa por medio de un pronombre. En (15) esta correferencialidad ocurre entre el nombre propio DARÍIN y el pronombre personal SHI.

\section{Relativas con antecedentes no referenciales y relativas sin antecedente}

Cuando el núcleo nominal tiene un carácter no referencial, es decir, cuando el núcleo nominal no hace referencia a una entidad específica, entonces este núcleo solamente puede ser modificado por una cláusula relativa restrictiva.

(16) A niid sombádi [we fi elp mi].

Yo necesitar alguien REL COM ayudar me

Necesito alguien que me ayude.

(17) A waan sombádi [uu fi elp mi kliin mi kluoz dem].

Yo querer alguien REL COM ayudar me limpiar mi ropa PLU

Quiero a alguien que me ayude a lavar la ropa. 
(18) A bring chainí fuud fi aal [uu waant].

Yo traer china comida para todo REL querer

Traje comida china para todo el que quiera.

(19) Éniwan [kom liet], naa paas.

Cualquiera venir tarde NEG-IRR pasar

Cualquiera que llegue tarde, no va a pasar.

En las oraciones anteriores (16-19), el núcleo nominal no referencial está expresado por pronombres indefinidos.

Además, las cláusulas relativas pueden modificar pronombres personales. En estos casos, por el contrario, la referencialidad es muy alta. Por eso, las relativas correspondientes son más bien no restrictivas.

(20) Dem [uu kom], a mi fren.

Ellos REL venir COP-ECU mi amigo

(Ellos,) los que llegaron, son mis amigos.

(21) Unu [we laik baal giem] guláng.

Ustedes REL gustar bola juego ir

Ustedes [que les gusta jugar bola], vayan.

(22) Wi [dat nuo nótin], afi go uom.

Nosotros REL saber nada MOD-DEB ir hogar

Nosotros, que no sabemos nada, tenemos que irnos para la casa.

(23) Mi [we work so much], waan res.

Yo REL trabajar así mucho querer descanso

Yo que trabajo tanto, necesito un descanso.

Por otro lado, sin embargo, la oración (24) contiene una cláusula restrictiva que modifica un pronombre indefinido con un baja referencialidad UU.

(24) Uu [uu duon go bai di bank nou] naa get móni til mónde.

Quien REL no ir por el banco ahora NEG-IRR obtener dinero hasta lunes Aquellos que no van al banco hoy no tendrán plata hasta el lunes.

Es notable que pronombre indefinido UU es prácticamente idéntico al pronombre relativo de la cláusula subordinada. Ambos claramente están relacionados diacrónicamente entre sí y con respecto del pronombre interrogativo UU.

La oración anterior es muy semejante tanto formal como semánticamente a las cláusulas que carecen de un antecedente explícito. Esta últimas pueden modificar tanto un antecedente específico [(25) y (26)] como un antecedente no específico (27). 
(25) [Uu brok dong di wínda], a uu fi fiks i.

REL quebrar abajo la ventana, FOC REL MOD-DEB arreglar ello

El que quebró la ventana, es el que debe arreglarla.

(26) [Uu ai bilíiv woz kómin], a no yu, a im.

REL yo creer ANT venir-PRO FOC no usted FOC él

El que yo creía que iba a venir, no era usted, sino él.

(27) [Uu a go shap] bring sigyáar.

REL PRO ir tienda traer cigarro

El que vaya a la pulpería me trae cigarros.

\section{Elementos de la oración relativa}

Se pueden reconocer tres elementos que están implicados en la formación de una cláusula relativa (cf. Payne 1997:325-6):

\section{a. El antecedente de la cláusula relativa}

El antecedente es la frase nominal en cláusula principal que está modificada por la oración relativa.

(28) Di man [uu dem a taak bout] a no fi mi ózban.

El hombre REL ellos PRO hablar sobre COP-ECU no para mí esposo

El hombre del que están hablando no es mi marido.

(29) Di tiim [we win dis ya giem] a go truu.

El equipo REL ganar este aquí juego PRO pasar

El equipo que gane este juego va a clasificar.

Como se ha dicho antes, también es posible que aparezcan oraciones relativas sin antecedente.

(30) $\varnothing$ [uu no work], duon iit.

$\varnothing$ REL no trabajar no comer

El que no trabaja, no come.

Como se ha dicho anteriormente, en esta lengua, el antecedente suele encontrarse inmediatamente antes de la cláusula relativa. Sin embargo, en algunas ocasiones, la cláusula relativa no se encuentra inmediatamente después del antecedente en la frase nominal sujeto, sino que lo hace al final del predicado.

(31) Di bed brok op [we dem did a jomp pan]

La cama quebrar REL ellos ANT PRO brincar en

La cama donde estuvieron brincando se quebró. 
(32) Dat gyal príti [uu mi did waan daans wid]

Esa muchacha ser-bonita REL yo ANT querer bailar con

Esa muchacha con la que quería bailar es bonita.

\section{b. La frase nominal relativizada}

La frase nominal relativizada es el elemento dentro de la cláusula relativa que hace referencia a la frase nominal antecedente.

(33) Di gorlfrén [we mi av] véri príti.

La novia REL yo tener muy ser-bonita

La novia que tengo es muy bonita.

(34) Di fren [uu yu shuo mi] shi príti.

La amiga REL usted mostrar me ella ser-bonita

La amiga que usted me presentó es bonita.

Las frase nominales relativizadas que son correferencial con el antecendente (DI GORLFRÉN, DI FREN) están representadas por medio de los pronombres relativos WE y UU, respectivamente, en las oraciones anteriores.

\section{c. El relativizador}

Es el elemento que relaciona la cláusula relativa con la cláusula principal y la subordina a ella. El relativizador puede ser un pronombre relativo: UU, WE, WA.

(35) Láisa, [uu Íirik a ded fa], lov neks wan.

L. REL I. PRO morir por amar otro uno.

Lisa, por quien Erick se está muriendo, quiere a otro.

(36) Yu, [we se yu a mi fren], du dat?

Usted REL decir usted COP-ECU mi amigo hacer eso

Usted que decía que era mi amigo, ¿hizo eso?

Di pícho [wa wi go si] di gud, man.

La película REL nosotros ir ver ANT ser-buena, hombre

La película que fuimos a ver estaba buenísima.

Estos relativizadores son pronombres porque expresan ciertas características semánticas de la frase nominal relativizada. Es decir, tienen una función anafórica. El pronombre uU tiene como antecedentes frases nominales que se refieren a seres animados, prototípicamente humanos. Por otro lado, el pronombre wA se utiliza con antecedentes que se refieren a objetos inanimados.Además, la función gramatical de este pronombre relativo es siempre de objeto directo. Por su parte, el pronombre wE puede usarse con antecedentes que hacen referencia tanto a seres animados como inanimados. 
También, es importante señalar que estos tres pronombres relativos están relacionados diacrónicamente con los pronombres interrogativos WA qué, we dónde, qué y uU quién, los cuales portan un tono alto a diferencia de los relativos que llevan un tono bajo.

Con cláusulas relativas sin antecedente, la función gramatical prototípica de los pronombre relativos UU y wA es la de sujeto y la de objeto directo de la oración subordinada respectivamente. Por su parte, la interpretación semántica prototípica del pronombre relativo wE es de un objeto oblicuo más que de un objeto directo.

(38) [Uu stanóp fors tumára], wíek mi op.

REL levantarse primero mañana despertar me

El que se levanta mañana primero, me despierta.

(39) [Wa mi ríili waan] a stie uom.

REL yo realmente querer COP quedar hogar

Lo que quiero realmente es quedarme en la casa.

(40) [We a bilíiv wi a go], a no de, a ya.

REL yo creer nosotros PRO ir FOC no ahí, FOC aquí

Donde yo creo que vamos no es allá, es acá.

Otros pronombres relativos que aparecen en esta lengua, pero menos frecuentemente, son WICH el cual, OU como y WAI por el que.

(41) Di tíebl [an wich im put it] waz dórti.

La mesa en la cual él poner eso ANT estar-sucia

La mesa en la que lo puso estaba sucia.

(42) A no laik di wie [ou im did it] atáal.

Yo no gustar la manera como él hacer(pasado) eso del-todo

No me gustó para nada la forma como lo hizo.

(43) Di ríizin [wai im kom] iz impúortant.

La razón por la que él venir COP ser-importante

La razón por la que vino es importante.

Por lo visto, el uso de estos pronombres se debe a la influencia del inglés estándar como lengua de superestrato. Esto parece evidente en los ejemplos anteriores, en los que se encuentran varios rasgos propios de los mesolectos, como el marcador de tiempo anterior WAZ (cf. basilecto DI), la cópula IZ (cf. basilecto A) e, incluso, una forma marcada para el pasado del verbo hacer DID (cf. basilecto DU).

También, estos pronombres relativos están relacionados diacrónicamente con los pronombres interrogativos WICH, OU y WAI, de los cuales se distinguen solamente por el tono.

Por otro lado, el relativizador también puede ser la conjunción subordinante DAT. Se trata de un nexo subordinador sin contenido semántico, sin valor anafórico respecto del antecedente. Es interesante señalar que este relativizador es idéntico a un completizador que introduce oraciones subordinadas completivas en esta lengua. 
(44) A laik stúori [dat yu tel mi laas nait].

Yo gustar cuento REL vos contar me pasada noche

Me gustó la historia que me contaste anoche.

(45) Frank se [dat you mosa kaal im].

F. decir COM vos MOD-DEB llamar lo

Frank dijo que tenías que haberlo llamado.

La oración (44) contiene una cláusula relativa, mientras que (45) presenta una subordinada completiva.

La diferencia entre los relativizadores que son pronombres relativos y los relativizadores que son conjunciones subordinantes es que los primeros representan la frase nominal relativizada en la cláusula relativa, mientras que los últimos no lo hacen del todo.

\section{Formas de expresar la frase nominal relativizada}

Aunque la frase nominal relativizada establece una referencia anafórica con el antecedente, este y la frase nominal relativizada pueden cumplir distintas funciones gramaticales en las cláusulas donde aparecen. Esta situación se conoce como el problema de la recuperabilidad del caso (Givón 1990: 651). Las lenguas del mundo utilizan distintas estrategias para representar la función gramatical o el caso de la frase nominal relativizada.

En el inglés criollo de Costa Rica, se utilizan tres estrategias diferentes.

\section{a. La estrategia del espacio vacío}

Esta estrategia consiste en representar la frase nominal relativizada por medio de un espacio vacío. Tanto el contexto lingüístico como el orden de los constituyentes aseguran al oyente la recuperabilidad del caso que desempeña la frase nominal relativizada. Este tipo de estrategia es común en lenguas con un orden de los constituyentes rígido, como es el caso precisamente del criollo inglés del Costa Rica, que es del tipo SVO (cf. Portilla 1998:168).

(46) A wan pus $[\varnothing$ ron paas de] a sí.

FOC un gato correr pasar ahí yo ver

Lo que vi fue un gato que salió corriendo por ahí.

(47) Dis ya [yu a si Ø] no gud atáal.

Ese aquí usted PRO ver no ser-bueno del-todo

Ese que usted está viendo no sirve para nada.

(48) Di píipl [mi a luk fa Ø] néva de.

La gente yo PRO mirar por nunca COP-EXI

La gente que ando buscando nunca está. 
En (46) la frase nominal relativizada WAN PUS cumple la función de sujeto, ya que es el único argumento de la cláusula relativa. En (47) la frase nominal relativizada DIS YA funciona como objeto directo, ya que la posición de sujeto ya está ocupada por el pronombre YU. Lo mismo sucede en (48), donde la posición del sujeto está tomada por el pronombre MI. La frase nominal relativizada funciona como un objeto oblicuo, que estaría precedido por la preposición FA.

En todos los casos anteriores, la cláusula relativa es introducida sin ningún relativizador. Sin embargo, también es común que se utilice el relativizador DAT junto con la estrategia del espacio vacío.

(49) Dem [dat $\varnothing \mathrm{kom}]$ a fi mi fren.

Ellos REL venir COP-ECU para mí amigo

Esos que llegaron son mis amigos.

(50) Dem stap di pícho [dat wi waz a go si Ø].

Ellos parar la película REL nosotros ANT PRO ir ver

Quitaron la película que íbamos a ver.

(51) Di man [dat mi komplien tu $\varnothing]$ a no mi pupá.

El hombre REL yo quejar a COP-ECU no mi papá

El hombre con quien yo me quejo no es mi papá.

La frase nominal relativizada ocupa la posición del sujeto en (49), la de objeto directo en (50) y la de objeto oblicuo en (51). Los casos están representados por medio de la estrategia del espacio vacío. Todas las cláusulas relativas están introducidas por el relativizador DAT.

Es importante mencionar que una de las funciones del uso del relativizador DAT junto con la estrategia del espacio vacío es señalar que la predicación que sigue al antecedente no es parte de la oración principal, sino que está subordinada a este. Esto aparece claro en (49) cuya variante sin el relativizador (52) da como resultado la coordinación de dos oraciones. Es decir, la interpretación de normal de la relación gramatical de las oraciones es de parataxis en lugar de hipotaxis.
Dem kom. A fi mi fren.
Ellos venir. FOC ${ }^{4}$ para mí amigo
Ya llegaron. Son mis amigos

La misma situación ocurre en el caso de las oraciones (53) en una relación hipotaxis, y (54) en una de parataxis.

(53) Dam badaríeshan úman [dat shi órin mi]!

Maldición fastidio mujer REL ella apurar-PRO me

¡Qué fastidiosa mujer que me está apurando!

(54) Dam badaríeshan úman! shi órin mi.

Maldición fastidio mujer. Ella apurar-PRO me

¿Qué fastidiosa mujer! Me está apurando 


\section{b. Estrategia del uso de pronombres relativos}

El uso de pronombres relativos es la estrategia más común usada en esta lengua para representar la frase nominal relativizada en la cláusula relativa.

Como se ha dicho, los pronombres relativos que aparecen principalmente en esta lengua son we, uU y wa, así como wich, ou y waI.

Como relativizadores, los pronombres relativos relacionan la cláusula subordinada con el antecedente en la cláusula principal. Además, pueden expresan ciertos rasgos de la identidad del antecente. Uu se utiliza con seres animados, especialmente humanos. WA es usado cuando se trata de objetos inanimados.

(55) Diev, uu nuo shi a kom, shot op.

D. REL saber él PRO venir callar

Dave, quien sabe que ella va a llegar, se quedó callado.

(56) Di ous [ina wa mi liv] smaal.

La casa en REL vivir ser-pequeña

La casa en la que vivo es pequeña.

Hay que mencionar que la función gramatical que desempeñan los pronombres relativos es claramente recuperable tanto por el contexto como por la semántica de los mismos pronombres relativos. Es muy factible que el pronombre UU, que representa seres humanos, desempeñe un rol más central en la cláusula, como sujeto. A su vez, el pronombre wA, usado para representar objetos, juega un rol menos central como objeto directo u oblicuo.

Por otro lado, también es normal que las preposiciones no precedan a los pronombres relativos en las cláusulas subordinadas, como se nota en (57), una variante de (56) (cf. también 34).
Di ous [wa mi liv ina] smaal.
La casa REL yo vivir en ser-pequeña
La casa en la que vivo es pequeña.

Por supuesto, el pronombre uU puede desempeñar otras funciones gramaticales diferentes del sujeto. El pronombre WE puede desempeñar todas las funciones gramaticales posibles.

\section{c. La estrategia de retención de pronombres}

Esta estrategia consiste en utilizar un pronombre personal o un pronombre posesivo dentro de la cláusula relativa que concuerde con el antecedente ${ }^{5}$.

(58) Ríini, [uu im son biga dan fi mi], nak mi duor kom tel mi dat mi son lik i. R. REL su hijo mayor que para mí tocar mi puerta venir decir me COM mi hijo pegar le

Renée, cuyo hijo es mayor que el mío, me tocó la puerta para decirme que mi hijo le pegó. 
En la oración anterior, la frase nominal relativizada (RínI) está representada tanto por el pronombre relativo UU como por pronombre posesivo $\mathrm{IM}^{6}$ en la cláusula relativa.

Se presentan a continuación algunos ejemplos de uso de pronombres personales que representan la frase nominal relativizada en la cláusula relativa.

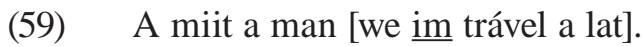

Yo conocer un hombre REL él viajar un montón

Conocí a un hombre que ha viajado mucho.

(60) A bai di soun [we mi di si it pan luo prais]

Yo comprar el sonido REL yo ANT ver ello en bajo precio

Me compré el equipo de sonido que había visto rebajado.

(61) Di yaad[we mi waak truu it] fens op.

El patio REL yo pasar a-través-de ello

Cercaron el patio por donde yo pasaba.

En (59) el pronombre personal que representa la frase nominal relativizada desempeña la función de sujeto; en (60) la función de objeto directo; y en (61) la función de objeto oblicuo. En los tres casos, la frase nominal relativizada también está representada por el pronombre WE.

\section{Relaciones gramaticales relativizadas}

Keenan \& Comrie (1979) postularon la existencia de una jerarquía de accesibilidad de las funciones o relaciones gramaticales que pueden ser relativizadas en una lengua. Esta jerarquía predice el siguiente ordenamiento: SUJETO > OBJETO DIRECTO > OBJETO INDIRECTO > OBJETO OBLICUO > GENITIVO > OBJETO DE COMPARACIÓN.

De acuerdo con esta jerarquía, si una relación gramatical a la derecha se puede relativizar, entonces deberán hacerlo todas las relaciones gramaticales que se encuentre a la izquierda. En este sentido, es más natural que se relativicen las relaciones gramaticales de sujeto, objeto directo y objeto indirecto, a que lo hagan las relaciones de genitivo o de objeto de comparación.

El inglés criollo de Costa Rica permite relativizar prácticamente todas las relaciones gramaticales mencionadas, independientemente de la estrategia de representación de la frase nominal relativizada.

Cuando se emplea la estrategia de uso de pronombres relativos, se pueden relativizar todas las relaciones gramaticales de la jerarquía.

(62) Di gyal [we a wiet fi mi] til de de.

(Sujeto)

La muchacha REL PRO esperar por mí todavía COP-EXI ahí

La muchacha que me está esperando todavía está ahí.

(63) Di gyal [we mi waan kaal laas nait] lain bad. (Objeto directo)

La muchacha REL yo querer llamar pasada noche línea estar-mala

La muchacha a la que quería llamar anoche tenía la línea mala. 
(64) Di gyal [we mi uo som móni] a biáin mi.

(Objeto indirecto)

La muchacha REL yo deber algún dinero COP venir detrás-de mí

La muchacha a la que le debo dinero anda detrás de mí.

(65) Di gyal [we mi a wiet pan] no shuo op. (Objeto oblicuo)

La muchacha REL yo PRO esperar por no aparecer

La muchacha que estuve esperando no se apareció.

(66) Di gyal [we di bréda nuo yu] outsáid de. (Posesivo)

La muchacha REL el hermano conocer te afuera COP-EXi

La muchacha, cuyo hermano te conoce, está ahí afuera

(67) Di gyal [we mi dáata bíga dan] bos agén. (Objeto de comparación)

La muchacha REL mi hija mayor que reventar otra-vez

La muchacha que mi hija es mayor que ella se volvió quedar.

Cuando se utiliza la estrategia del espacio vacío y el uso del relativizador DAT, también pueden ser relativizadas todas las relaciones gramaticales que contempla la jerarquía.

Di gyal [dat $\varnothing$ a wiet fi mi] til de de. $\quad$ (Sujeto)

La muchacha REL PRO esperar por mí todavía COP-EXI ahí

La muchacha que me está esperando todavía está ahí. [...]

(69) Di gyal [dat $\varnothing$ di bréda nuo yu] outsáid de.

La muchacha REL el hermano conocer te afuera COP-EXi

La muchacha, cuyo hermano te conoce, está ahí afuera

Me llamó la muchacha de ojos bonitos.

(70) Di gyal [dat mi dáata bíga dan Ø] bos agén. (Objeto de comparación)

La muchacha REL mi hija mayor que reventar otra-vez

La muchacha que mi hija es mayor que ella se volvió quedar.

Sin embargo, cuando se emplea la estrategia del espacio vacío pero sin relativizador, resulta agramatical la relativización de la relación gramatical de posesivo.

(71) Di gyal [Ø a wiet fi mi] til de de.

(Sujeto)

La muchacha REL PRO esperar por mí todavía COP-EXI ahí

La muchacha que me está esperando todavía está ahí.

(72) Di gyal [mi waan kaal $\varnothing$ laas nait] lain bad.

(Objeto directo)

La muchacha REL yo querer llamar pasada noche línea estar-mala

La muchacha a la que quería llamar anoche tenía la línea mala.

(73) Di gyal [mi uo Ø som móni] a biáin mi. (Objeto indirecto)

La muchacha REL yo deber algún dinero COP venir detrás-de mí

La muchacha a la que le debo dinero anda detrás de mí. 
(74) Di gyal [mi a wiet pan Ø] no shuo op.

(Objeto oblicuo)

La muchacha REL yo PRO esperar por no aparecer

La muchacha que estuve esperando no se apareció.

* Di gyal [ $\varnothing$ di bréda nuo yu] outsáid de. (Posesivo)

La muchacha el hermano conocer te afuera COP-EXi

La muchacha, cuyo hermano te conoce, está ahí afuera

Di gyal [mi dáata bíga dan Ø] bos agén.

(Objeto de comparación)

La muchacha REL mi hija mayor que reventar otra-vez

La muchacha que mi hija es mayor que ella se volvió quedar.

Otro ejemplo de la agramaticalidad de la relativización de la relación de posesivo con la estrategia del espacio vacío sin relativizador aparece en (75), en contraste con (76) y (77) en las que utilizan relativizadores.

(77) * Dat daag [ $\varnothing$ tiit big] bad. (Estrategia del espacio vacío sin relativizador) Ese perro diente ser-grande ser-malo Ese perro con dientotes es bravo.

(78) Dat daag [we tiit big] bad. (Estrategia del uso de pronombre relativo) Ese perro REL diente ser-grande malo Ese perro con dientotes es bravo.

(79) Dat daag [dat $\varnothing$ tiit big] bad. (Estrategia del espacio vacío con relativizador) Ese perro REL diente ser-grande malo Ese perro con dientotes es bravo.

De manera general, Payne (1997: 335) explica esta asimetría aduciendo que las estrategias de relativización más explícitas (como en este caso el uso de un pronombre relativo o de un relativizador) son más proclives a relativizar mayor cantidad de relaciones gramaticales que las estrategias menos explícitas (como la estrategia del espacio vacío).

Por otro lado, es notable que con la estrategia del espacio vacío se pueda relativizar la relación de objeto de comparación a pesar de que no pueda hacerlo la de genitivo, pues esto contradice la jerarquía propuesta por Keenan \& Comrie (1979).

\section{Conclusiones}

Las cláusulas relativas en el inglés criollo de Costa Rica presentan las mismas características tipológicas de las lenguas SVO. Estas aparecen después del antecedente que modifican.

Desde el punto de vista pragmático-discursivo, existen en esta lengua tres tipos de cláusulas relativas: restrictivas, no restrictivas y sin antecedente explícito. 
Se utilizan tres estrategias para representar la frase nominal relativizada en la cláusula relativa: la estrategia del espacio vacío, del uso de pronombres relativos y de retención de pronombres. El uso de pronombres relativos es la estrategia más común utilizada en esta lengua.

El inglés criollo de Costa Rica permite relativizar prácticamente todas las relaciones gramaticales posibles, independientemente de la estrategia de representación de la frase nominal relativizada.

\section{Abreviaturas}

$\begin{array}{ll}\text { ANT } & \text { Tiempo anterior } \\ \text { COP-ECU } & \text { Cópula ecuativa } \\ \text { COP-EXI } & \text { Cópula existencial } \\ \text { DUR } & \text { Aspecto durativo } \\ \text { FOC } & \text { Marcador de foco o contraste } \\ \text { MOD-DEB } & \text { Modal deóntico de deber } \\ \text { NEG-IRR } & \text { Negación de modo irrealis } \\ \text { PLU } & \text { Plural } \\ \text { PRO } & \text { Aspecto progresivo } \\ \text { REL } & \text { Relativizador }\end{array}$

\section{Notas}

1. En esta oración, se ha aplicado una operación de anteposición (fronting) del objeto directo, la cual tiene una función topicalizadora.

2. El tiempo anterior (ANT) indica que el evento referido por el verbo ocurrió en un momento anterior al momento que se halla en foco en el discurso. Con verbos estativos, este corresponde a un tiempo pasado del español; pero, con verbos dinámicos, corresponde por lo general al pluscuamperfecto del español. Cf. Portilla 1997: 164.

3. Sankoff \& Brown (1976) hallan una construcción idéntica en el tok pisin, que es un pidgin extendido que se habla profusamente en Nueva Guinea. Estos autores consideran que el demostrativo YA funciona como un verdadero relativizador, ya que introduce siempre la cláusula no restrictiva. Sin embargo, por lo menos en el caso del criollo inglés de Costa Rica, habría que señalar que, en cuanto a la entonación, claramente el demostrativo forma parte de la cláusula principal y no de la subordinada.

4. Esta interpretación de (51) está reforzada por el hecho de que la cópula ecuativa es idéntica al marcador de foco.

5. En las variedades más basilectales, los pronombres personales son idénticos a los pronombres posesivos.

6. En un uso muy basilectal, como en este caso, en la tercera persona no se hace la distinción entre masculino y femenino. 


\section{Bibliografía}

Givón, T. 1984. Syntax. Volumen 1. Amsterdam: Benjamins.

Givón, T. 1990. Syntax. Volumen 2. Amsterdam: Benjamins.

Keenan, E. \& B. Comrie. 1979. "Data on the noun phrase accesibility hierarchy". Language 55: $332: 52$.

Payne, T. 1997. Describing morphosintax. Cambridge: Cambridge University Press.

Portilla, M. 1997. "Tiempo, aspecto y modo en el criollo inglés de Costa Rica". Revista de

Portilla, M. Filología y Lingüística de la Universidad de Costa Rica. 23 (2): 161-72.

Portilla, M. 1998. "Operaciones de reducción de valencia en el criollo inglés de Costa Rica". Revista de Filología y Lingüística de la Universidad de Costa Rica. 24 (2): 167.

Romaine, S. 1988. Pidgin and creole languages. Londres \& Nueva York: Longman.

Sankoff, G \& P. Brown. 1976. "The origins of syntax in discourse: the case of Tok Pisin relatives”. Language 52: 631: 66. 